\title{
Single Mode, polarisation independent waveguides in silicon-on-insulator
}

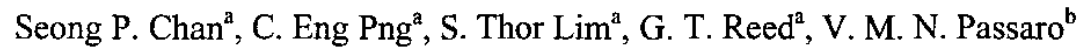

${ }^{a}$ School of Electronics and Physical Sciences, University of Surrey, Guildford, GU2 7XH, UK

${ }^{b}$ Dipartimento di Elettrotecnica ed Elettronica, Politecnico di Bari, Via E. Orabona 4, 70125 Bari, Italy.

\begin{abstract}
The conditions for single mode and zero birefringence for small and deeply etched SOI waveguides are presented and discussed. It is difficult to satisfy both conditions simultaneously.
\end{abstract}

Discussion: There is a strong trend towards fabrication of smaller photonic devices to improve device performance and cost. However, the polarisation dependence and single mode conditions have not been discussed in sufficient detail for smaller waveguide dimensions. We have used the semivectorial beam propagation method

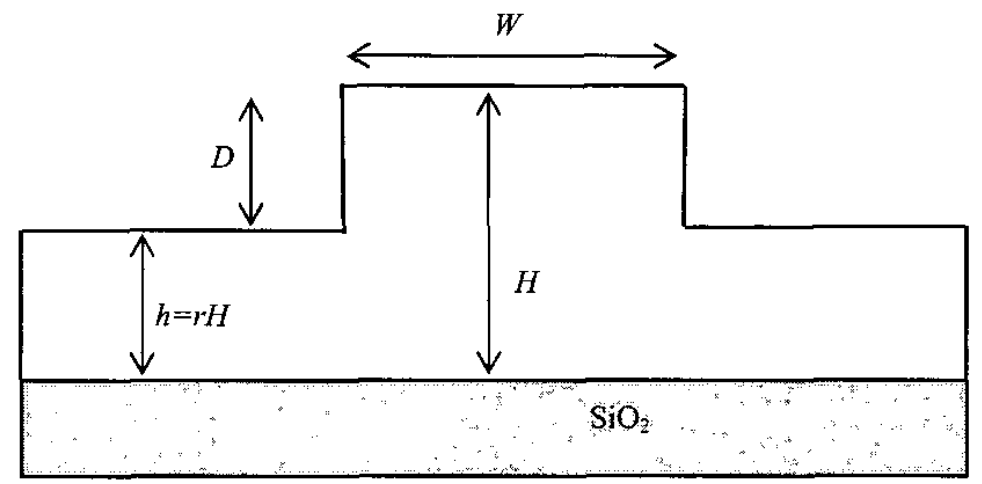

Figure I SOI Rib Waveguide (BPM) to analyse the waveguide structure in figure 1 for single mode propagation and zero birefringence both in TE and TM-like modes. The result for the single mode condition was compared with a finite-difference vector solver and the scalar approximation method; the simulation data are currently being compared with the more accurate finite difference time domain (FDTD) method. Soref et al. [1] first derived an expression for the single-mode condition (SMC) in relatively large rib waveguides in which parameter $r$ (see figure 1) of $0.5<r<1.0$ was imposed on the änalysis. $W$ and $H$ are also defined in figure 1 :

$\frac{W}{H} \leq 0.3+\frac{r}{\sqrt{1-r^{2}}}$.

The analytical Effective Index Method (EIM) by Pogossian et al. [2] suggested that more stringent criteria needed to be imposed for single mode operation, resulting in a modified equation:

$$
\frac{W}{H} \leq \frac{r}{\sqrt{1-r^{2}}}
$$

Subsequently Powell [3] made a more general analysis for rib waveguides with vertical or angled sidewalls [3], finding a simple analytical expression for such waveguides that was in good agreement with the original work of Soref et al. [1] as well as a

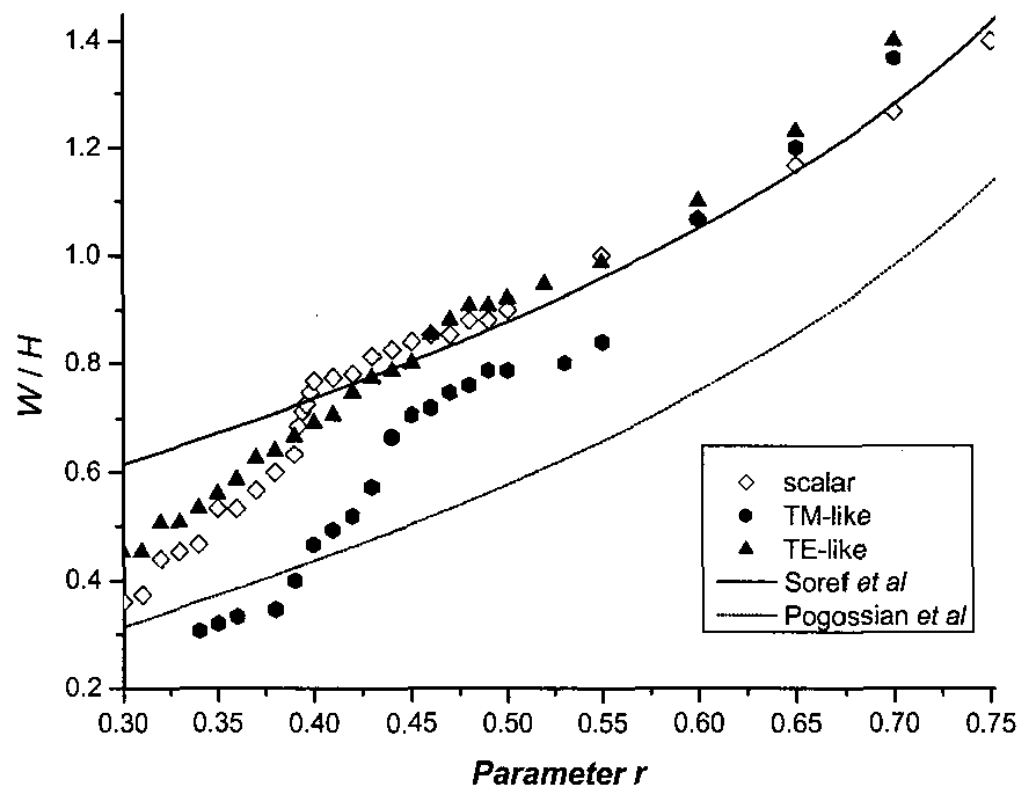

Figure 2 Comparison of various methods used to compute the single mode cut-off dimensions for rib waveguide height of $1.50 \mathrm{\mu m}$

simple scalar approximation that was also used in his analysis. He showed that the scalar approximation is sufficiently accurate for large rib waveguide with shallow etch depth (large $r$ ), where the mode profiles is similar for both polarisations. Nonetheless, the validity of these equations when applied to small waveguides and deeply etch 
rib waveguides has not been addressed sufficiently. The degree of influence of etch depth on waveguide performance also becomes apparent when small waveguides are designed for zero birefringence $[4,5]$. It is usually desirable to be able to satisfy both the single mode and the zero birefringence conditions to improve device performance in small SOI rib waveguides.

The single mode simulation was setup using the waveguide structure shown in Fig. 1, which is a rib waveguide of silicon $\left(n_{\mathrm{g}}=3.477\right)$ on silica $\left(n_{\mathrm{s}}=1.444\right)$ and has an upper cladding that is air $\left(n_{\mathrm{c}}=1\right)$. We assumed there is no coupling between the TE-like and TM-like fields and most of the field energy is in one polarisation (the major component). Our initial investigation was for a waveguide height of $1.50 \mu \mathrm{m}$ and operating wavelength of $\lambda=1550 \mathrm{~nm}$. The waveguide height $(H)$, etch depth $(D)$, and slab height $(h)$ of the waveguide were kept constant while increasing the waveguide width in increments of $0.01 \mu \mathrm{m}$ to find the single-mode/multimode boundary. The iteration of the simulation is repeated with different values of the slab height to waveguide height ratio, parameter $r$. The process is to gradually increase the waveguide width until a second mode propagates, thereby determining the maximum waveguide width to sustain single mode operation. In Figure 2, the results of mode cut-off using the semi-vectorial BPM approach were compared with the scalar approximation (Powell) and results from Soref and Pogossian whose results are extrapolated to include the range $r<0.5$. One can observe the results for the scalar approximation and TE-like result are very similar for large range of values of parameter $r$. This scalar approximation result is also in surprisingly close agreement with [1] and [3] for values of parameter $r$ less than approximately 0.40 , where small rib waveguides with low width to height ratio are very polarisation dependent, hence the need for this analysis. However, the simulation suggested that the results of Pogossian et al. [2] are less useful. It will be shown later that the SMC for both polarisations converges for values of the parameter $r$ greater than approximately 0.55 , in good agreement with the original work of Soref et al. [1]. This confirms that the work of Soref et al. [1], or even the scalar approximation can be used as a basis of design of single mode waveguides for shallow etch rib waveguide $(r>0.5)$ with large cross section. However, the differences between the single mode conditions for different polarisations at smaller values of parameter $r$, particularly in small waveguides, are significant. This is because the optical confinement for such small waveguides is dominated by the boundary conditions, which are well known to be different for TE-like and TM-like modes. If we turn our attention to the design of birefringence free waveguides, we can then provide guidelines for achieving both single mode behaviour and polarisation independence in the waveguide.

The degree of influence of waveguide width and etch depth on waveguide birefringence for a given rib height is demonstrated further here. We used a rib waveguide height of $1.50 \mu \mathrm{m}$ for consistency with our analysis in figure 1 . We then evaluated the effective indices of the fundamental TE-like and TM-like modes. If we assume an operating wavelength of $1550 \mathrm{~nm}$, we can produce a graph of the variation of the TE/TM fundamental mode effective index difference for various etch depths $(D)$ and waveguide widths $(W)$. Figure 3 shows various curves, each for a different etch depth. The curves cross the zero birefringence axis when effective indices of both polarisation modes is the same, which indicates it is possible to produce birefringence free waveguides for some of the geometries and dimensions when a deeper etch depth is employed. All the data points which cross the zero birefringence axis are used in figure 4 in terms of $W / H$ ratio and parameter $r$, to plot a locus of the birefringence free condition for varying waveguide dimensions. This is used to show the relationship between single mode and birefringence free operation and

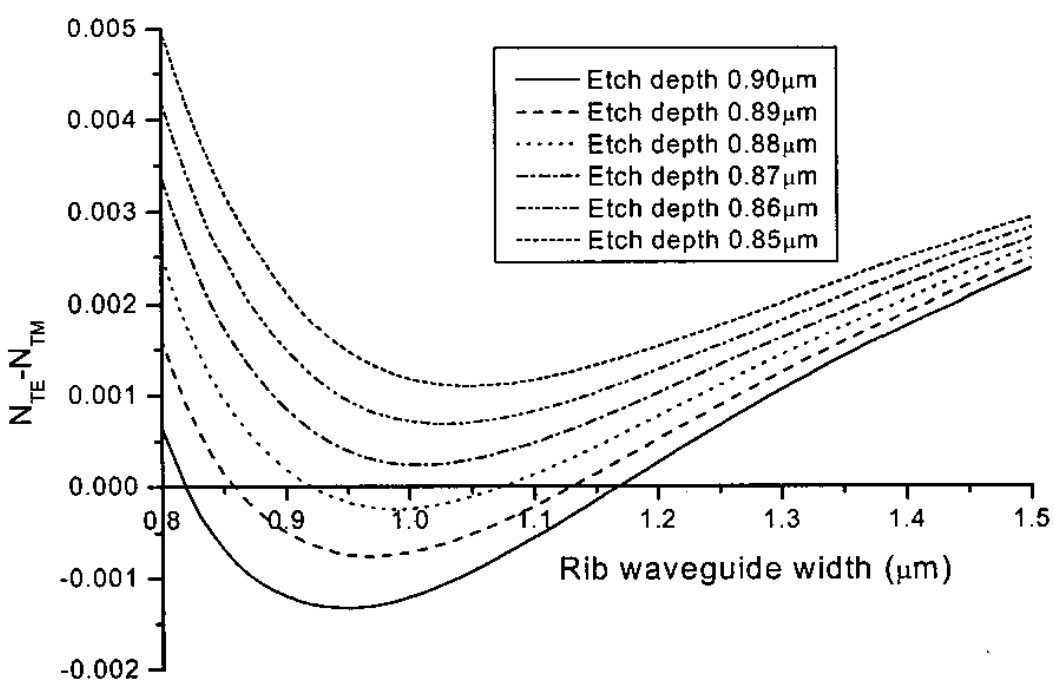

Figure 3 Effective index difference plotted against rib width for various etch depths 
the possibility of satisfying both conditions simultaneously. Similar data is included for a second waveguide height of $1.35 \mu \mathrm{m}$. Also included in figure 4 are trend lines for the single mode condition for TE-like and TM-like modes. It is clear from figures $4 \mathrm{a}$ and $4 \mathrm{~b}$ that it is difficult to achieve single mode operation at both polarisations whilst maintaining polarisation independence. It should be noted that the trend lines are approximate because they are the results of curve fitting, therefore for a specific design, data points should be used instead of the trend lines. In order to satisfy both the requirements of single mode operation and polarisation independence, we must choose a point on the birefringence free locus that is below the single mode boundary for both TE-like and TM-like modes. It is interesting to note that the intersection between the birefringence free locus and the TE-like graphs determines the limit of the waveguide width to guide only the fundamental TE mode. For instance, the maximum values of rib width are $0.98 \mu \mathrm{m}$ and $1.07 \mu \mathrm{m}$ for waveguide heights of $1.35 \mu \mathrm{m}$ and $1.50 \mu \mathrm{m}$ respectively, to satisfy the TE-like single mode condition and the birefringence free condition, but the birefringence free and TM-like single mode trend lines do not cross (although the scatter of the data about the trend line means that in some cases both conditions can be met). It should be noted that single mode condition is similar but not the same for different waveguide heights as illustrated in figures $4 \mathrm{a}$ and $4 \mathrm{~b}$. Both single mode behaviour for TE-like and TM-like converged approximately at parameter $r=0.55$, suggesting that scalar approximation can be used as a basis of designs greater than $r=0.55$ but trend lines are preferably for smaller values of parameter $r$.

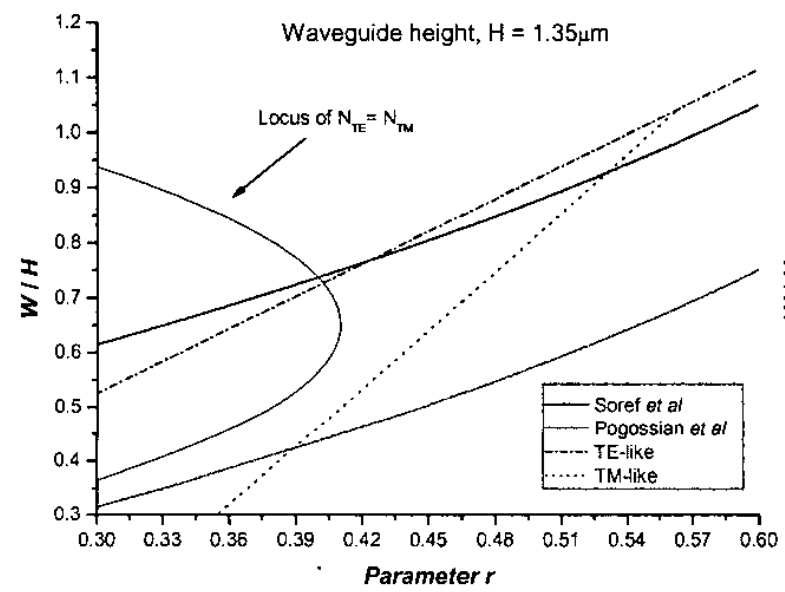

(a)

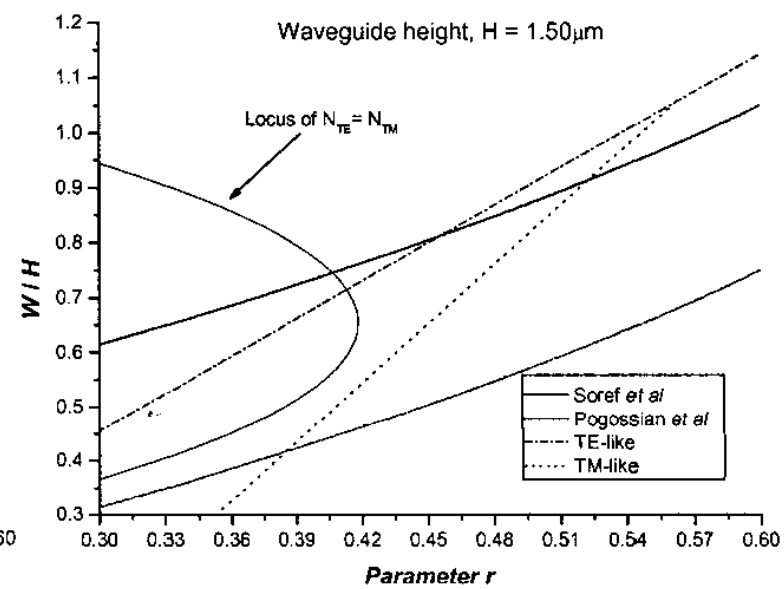

(b)

Figure 4 Trend lines for single mode cut-off dimensions and zero birefringence as a function of waveguide dimensions, for an operating wavelength of $\lambda=1550 \mathrm{~nm}$, and for waveguide heights of (a) $1.35 \mu \mathrm{m}$ and (b) $1.50 \mu \mathrm{m}$

\section{Conclusion:}

We have studied the design parameters of rib waveguide structure to satisfy both single mode and polarisation independence. The requirement of birefringence free waveguides can be fulfilled by using a deep etch depth when using small waveguides, but it is difficult to simultaneously satisfy the single mode conditions. Not surprisingly the scalar approximation is accurate enough to design single mode waveguide with large cross section for $r>0.5$, in agreement with results of Soref et al. [1] and Powell [3]. Hence individual design simulation should be used as a basis to design waveguides with smaller parameter of $r$ (deep etch) to achieve single mode and birefringence free operation simultaneously, or for less stringent requirements our trend lines are a useful guideline.

\section{References:}

[1] R. A. Soref, J. Schmidtchen and K. Petermann, "Large single-mode rib waveguides in Ge-Si and Si-on-SiO ${ }_{2}$, IEEE Journal of Quantum Electronics, Vol. 27, No.8 August 1991, pp. 1971-1974

[2] S. P. Pogossian, L. Vescan, and A. Vonsovici, "The single-mode condition for semiconductor rib waveguides with large cross section", Journal of Lightwave Technology, Vol. 16, No. 10, October 1998, pp. 1851-1853

[3] O. Powell, "Single-mode condition for silicon rib waveguides", Journal of Lightwave Technology, Vol. 20, No. 10, October 2002, pp. 1851-1855

[4] L. Vivien, S. Laval, B. Dumont, S. Lardenois, A. Koster, E. Cassan, "Polarization-independent single-mode rib waveguides on silicon-on-insulator for telecommunication wavelengths", Optics Communications, Vol. 210, September 2002, pp.43-49.

[5] S. P. Chan, V. M. N. Passaro, S. T. Lim, C. E. Png and G. T. Reed, "Characterization of integrated Bragg gratings on SOI rib waveguides", Proc. SPIE Int. Soc. Op. Eng., 5248, 273, 2003 